\title{
Network Coding Based Algorithm for WMN Scenario
}

\author{
Sehrish Shoaib and Muhammad Younus Javed
}

\begin{abstract}
Wireless Mesh Networks (WMN) provide significant benefits over existing wireless multi-hop networking paradigms, offering a suitable solution for various application scenarios. WMNs utilize legacy-based routing protocols for their communication. A set of innovative routing approaches have been studied. In this paper a classification of the most representative solutions in literature for WMNs is provided. A performance issue observed in WMNs is accumulation of data around the gateway nodes causing congestion and a degradation of output of the network. From the literature research conducted an algorithm is proposed.
\end{abstract}

Index Terms-Wireless mesh networks (WMN), network coding, algorithms.

\section{INTRODUCTION}

WMNs have become an emerging technology for the last mile network for mobile devices. For the purpose of internet connectivity; in WMNs the setup is made in such a way that a limited number of nodes act as connection points to access internet; such nodes are known as gateway nodes and maximum traffic arrives at this node so as to be able to provide services such as Http, Ftp and VoIP. WMNs provide us with effective and cheap networking with almost negligible infrastructure backing. Their applications vary from assisting the wireless office to disaster aid. However the in-built unpredictability of the wireless network and inter-reliant scheduling, routing and intrusion issues are a big timely challenge for the design of the network that performs well. One of the characteristic of a WMN is the large number of routes it provides to link a source node to its specified destination. In this way multiple routes are likely to give a better performance. However, this transmission of data packets across different routes leads to new issues of congestion and unfairness. A major cause for degradation in the output of WMN is congestion at the gateway links. Congestion is caused due to traffic that accumulates at the nearby gateway nodes. Congestion control is an issue that has been widely researched in the context of the internet framework [1]-[3], and Ad-hoc network framework [4]. However most work done in this area has been focused on the Transmission Control Protocol (TCP), and its variations. Network coding has lately appeared to be a new coding paradigm which has proved to show an extensive variety of claims for bringing improvement in the enactment of a WMN [5]. Network coding operates by allowing mixing of the

Manuscript received October 11, 2014; revised December 13, 2014.

Sehrish Shoaib is with the National University of Sciences and Technology, Islamambad, Pakistan (e-mail: sehrishmk@gmail.com).

Muhammad Younas Javed is with National University of Sciences and Technology, College of Electrical and Mechanical Engineering, Islamambad, Pakistan (e-mail: myjaved@ceme.nust.edu.pk). incoming data from multiple links at a transitional node for further transmission, as a result of which there is an improvement in the network performance regarding throughput. Therefore, Network Coding is focused; since it is a promising way out to be taken into consideration for improving the proficiency of a wireless network while reducing congestion within the network. Network coding is a new research field bringing change in the customary idea of routing, by not only forwarding data packets at the intermediate node rather also recombining some input packets into one or more output packets in order to obtain maximum information flow in the network. Network coding has improved the output in terms of throughput for peer to peer networks and satellite links. Network coding addresses the information aspect of network coding since it has its roots in information theory and coding theory. Analyzing the solutions for the purpose of reduction in redundant transfers of data over a network it can be deduced that network coding is an optimum solution for the congestion issue challenged in WMNs.

\section{Overview OF WiRELESS MESh NeTWORKS}

The concept of mesh networking can be understood via mesh topology and its implementation. A node is a communication endpoint capable of sending, receiving, or forwarding data in a communication network. If a network consists of $\mathrm{n}$ nodes then it is considered a mesh network if each node can connect to every other node. WMNs are regarded as a key technology that supports the combination of a wireless backbone with a wired infrastructure. Easily and effectively connecting entire cites $\&$ also providing internet to mobile nodes. Major characteristics of WMNs are its ability to organize and configure dynamically.

\section{A. Mesh Networking Defined}

WMNs are formed by semi-permanent self-organizing wireless routers connected in a partial mesh topology via wireless communication links, providing a backbone network service to mobile clients. In a WMN, a set of dedicated routers that form the strength of the network, provide communication support for mesh clients; this backbone is fixed and wireless in nature. The mesh topology and multi-hop communications provide redundant transmission paths and allow highly flexible network deployments in a WMN [6].

Characteristics of WMNs make it suitable for scenarios of home networking, campus networking, metropolitan networking and emergency networks in disaster zones [7]. Potentially, WMNs can support the principal of communication anytime, anywhere, in a timely, cost-effective way [8]. 
Definition: A WMN is a packet-switched system with a wireless support [9].

Hence,

- Topology for the backbone in WMN is fixed in nature and access point mobilization does not become an issue.

- Alterations to infrastructure can be done by removal or addition or failure of access points.

- Pure Ad-hoc networks are not regarded as WMNs.

\section{TAXONOMY FOR WMN ROUTING}

\section{A. Legacy Based Routing}

Due to the similarities of WMNs in terms of design with wired Mesh networks, a simple design approach is to adopt the routing paradigms traditionally conceived for wired networks. The assumption that wireless links can be replaced by wired links as point to point connections is implemented in such design approaches; this category of networking protocols is known as "legacy routing solutions". In legacy routing solutions the method for choice of route between a source-destination pair, is to pre-compute one or more minimum cost paths. Based on the classification of legacy based protocols, taxonomy for WMN routing protocols can be established as shown in Fig. 1.

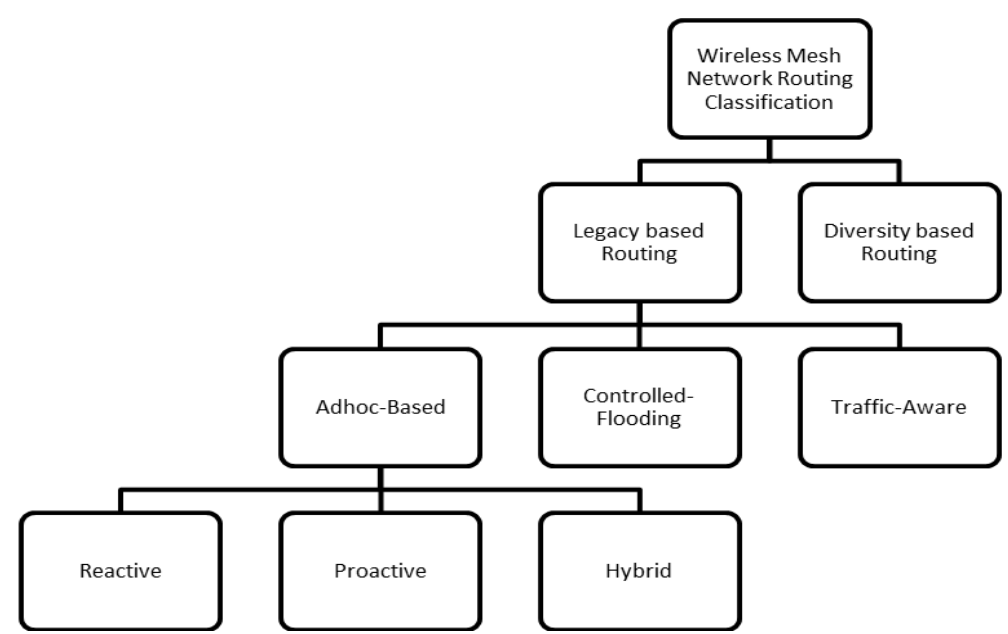

Fig. 1. Taxonomy of Legacy based routing protocols for WMNs

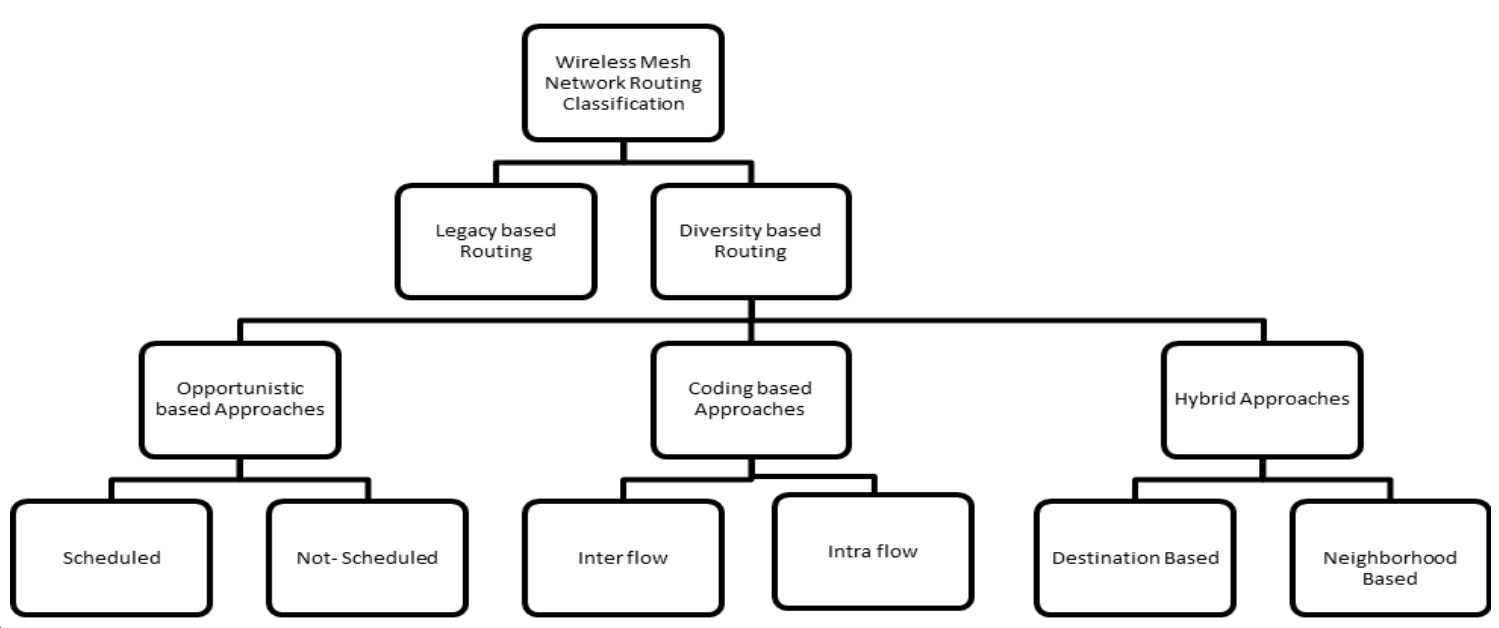

Fig. 2. Taxonomy of Diversity based routing protocols for WMNs.

\section{1) Adhoc based}

Due to the similarities that Mesh networks have with Ad-hoc networks, routing protocols implemented in Ad-hoc scenarios are used as a basis for the development of efficient routing protocols in mesh scenarios. Ad-hoc routing protocols are classified into three main categories; Reactive, Proactive and Hybrid. Reactive routing protocols are also known as on-demand. In the on demand method for routing, as the name implies the sender will search for a route to the destination node only when the data is ready for transmission. Proactive routing protocols are also known as table-driven. Such routing protocols maintain routing tables that have complete routing information about all the nodes existing in the network. Hybrid routing protocols combine features of both proactive and reactive ones. The topology is distributed into specified zones. Distribution of data within a zone is table driven (proactive) and for inter-communication between nodes of different zones, on-demand (reactive) routing protocol is implemented.

\section{2) Controlled-flooding protocols}

The basic idea behind controlled-flooding protocols is the decrease in control overhead. Scalability issues become a main factor while flooding the network with information about updates of routes. Two types of flooding approaches 
are used; repetition of flooding is based on distance from source router in temporal flooding. Nodes that are at a greater distance from source node receive more concise and less detailed information; in spatial flooding. Generally protocols adopt the temporal flooding technique. This is based on the assumption that maximum communication occurs between nodes that are near to each other and hence flooding the entire network decreases efficiency of the network.

TABLE I: EXAMPLES OF PROTOCOLS ACCORDING TO THE CATEGORIZATION CRITERIA DISCUSSED IN THIS PAPER

\begin{tabular}{|c|c|c|c|c|c|}
\hline \multicolumn{6}{|c|}{ Examples of Protocols } \\
\hline & Protocol & Categorization & Routing & Coding & ACK strategy \\
\hline AODV & $\begin{array}{l}\text { Ad-hoc on Demand } \\
\text { Distance Vector Protocol }\end{array}$ & Adhoc based & $\begin{array}{l}\text { On demand } \\
\text { route setup }\end{array}$ & No & Route Reply Messages \\
\hline DSR & Dynamic Source Routing & Adhoc based & $\begin{array}{l}\text { On demand } \\
\text { route setup }\end{array}$ & No & Route Reply Messages \\
\hline OLSR & $\begin{array}{l}\text { Optimizer Link State } \\
\text { Protocol }\end{array}$ & Adhoc based & Proactive & No & Route Reply Messages \\
\hline LQSR & $\begin{array}{l}\text { Link Quality Source } \\
\text { Routing Protocol }\end{array}$ & Adhoc based & $\begin{array}{l}\text { Hybrid based on } \\
\text { DSR }\end{array}$ & No & Route Reply Messages \\
\hline MR-LQSR & Multi Radio LQSR & Adhoc based & Hybrid & No & Route Reply Messages \\
\hline LOLS & $\begin{array}{l}\text { Localized On-Demand Link } \\
\text { State Protocol }\end{array}$ & Controlled flooding & $\begin{array}{l}\text { Legacy based } \\
\text { flooding }\end{array}$ & No & Route Reply Messages \\
\hline MMRP & $\begin{array}{l}\text { Mobile Mesh Routing } \\
\text { Protocol }\end{array}$ & Controlled flooding & $\begin{array}{l}\text { Legacy based } \\
\text { flooding }\end{array}$ & No & Route Reply Messages \\
\hline AODV-ST & $\begin{array}{l}\text { Ad-hoc On-Demand } \\
\text { Distance Vector-Spanning } \\
\text { Tree }\end{array}$ & Traffic Aware & $\begin{array}{l}\text { Maintenance of } \\
\text { routing table for } \\
\text { gateway node }\end{array}$ & No & Route Reply Messages \\
\hline ExOR & $\begin{array}{l}\text { Extremely Opportunistic } \\
\text { Routing protocol }\end{array}$ & $\begin{array}{l}\text { Opportunistic based } \\
\text { (Scheduled) }\end{array}$ & Opportunistic & No & Batch map \\
\hline ROMER & $\begin{array}{l}\text { Resilient Opportunistic } \\
\text { Mesh Routing protocol }\end{array}$ & $\begin{array}{l}\text { Opportunistic based } \\
\text { (Not Scheduled) }\end{array}$ & Opportunistic & No & - \\
\hline COPE & COPE & $\begin{array}{l}\text { Coding based } \\
\text { (Inter-flow) }\end{array}$ & $\begin{array}{l}\text { Legacy with } \\
\text { Pseudo } \\
\text { broadcast } \\
\text { transmissions }\end{array}$ & $\begin{array}{l}\text { Inter-flow XOR } \\
\text { with reception } \\
\text { reports/ } \\
\text { guessing }\end{array}$ & Link-layer ACKs \\
\hline $\mathrm{MC} 2$ & $\begin{array}{l}\text { Multipath Code Casting } \\
\text { protocol }\end{array}$ & $\begin{array}{l}\text { Coding based } \\
\text { (Intra-flow) }\end{array}$ & $\begin{array}{l}\text { Legacy } \\
\text { multipath with } \\
\text { broadcast } \\
\text { transmissions }\end{array}$ & $\begin{array}{l}\text { Intra-flow } \\
\text { Random Linear } \\
\text { Coding }\end{array}$ & $\begin{array}{l}\text { Hop-by-hop } \\
\text { overhearing, end to- } \\
\text { end } \\
\text { retransmissions }\end{array}$ \\
\hline MORE & $\begin{array}{l}\text { MAC-independent } \\
\text { Opportunistic Routing and } \\
\text { Encoding protocol }\end{array}$ & $\begin{array}{l}\text { Hybrid } \\
\text { (Destination based) }\end{array}$ & Opportunistic & $\begin{array}{l}\text { Intra-flow } \\
\text { Random Linear } \\
\text { Coding }\end{array}$ & End-to-end ACKs \\
\hline $\mathrm{XCOR}$ & $\mathrm{XCOR}$ & $\begin{array}{l}\text { Hybrid } \\
\text { (Neighborhood } \\
\text { based) }\end{array}$ & Opportunistic & $\begin{array}{l}\text { Inter-flow XOR } \\
\text { with reception } \\
\text { reports }\end{array}$ & Reception reports \\
\hline
\end{tabular}

\section{3) Traffic-aware or tree-based protocols}

A tree-like network topology is considered in traffic aware or tree based protocols. A common-case application for such protocols is backhaul access.

\section{B. Diversity Based Routing}

\section{1) Opportunistic routing}

Opportunistic routing schemes improve classical routing based on cooperative diversity schemes. Opportunistic routing algorithms as the name refers to utilize hop by hop mechanism in forwarding decisions, at every single hop the next node to which data is to forwarded to selected after gathering information about the set of nodes that have already received this data. Optimization of nodes selection, in order to forward data is hence implemented in this way. This technique differs from legacy routing in which the next hop is already pre decided. Therefore all such algorithms which make use of information gathered during transmission of a packet on its path in contrast to using a predetermined path can be classified under opportunistic algorithms.

The opportunistic-based routing concept is as follows:

- A node that overhears a data transmission will then be considered a part of the forwarding process.

- The choosing of the next forwarding nodes takes place only after a packet is received.

Two classes of opportunistic-based routing solutions are shown as in Fig. 2.

- Scheduled: In scheduled algorithms a set of possible forwarding nodes and the order in which the nodes are to transmit the data to its destination is pre-identified by the protocol.

- Not-scheduled: Not scheduled schemes differ from scheduled schemes in their design mechanism such that they do not follow strict scheduling amongst the possible candidate nodes.

\section{2) Coding based rouuting}

The concept of Network coding allows the data being transmitted in a network from various nodes to be combined at an intermediary node; henceforth improving the throughput of a network. The broadcast nature of a wireless network allows room for network coding methods to be incorporated, since nodes are able to overhear neighboring nodes transmissions, increasing the possibility of an individual node to combine data from various nodes.

\section{3) Hybrid routing}

In the hybrid routing approach we combine the opportunistic and coding methods in order to gain the 
advantages that both these methods provide.

Hybrid schemes are divided into two categories; one is destination based and neighborhood based. In destination based coding decisions are made keeping the destination node as top priority whereas in neighborhood based coding decisions are based on neighborhood nodes.

Examples of above discussed protocols are given in Table I.

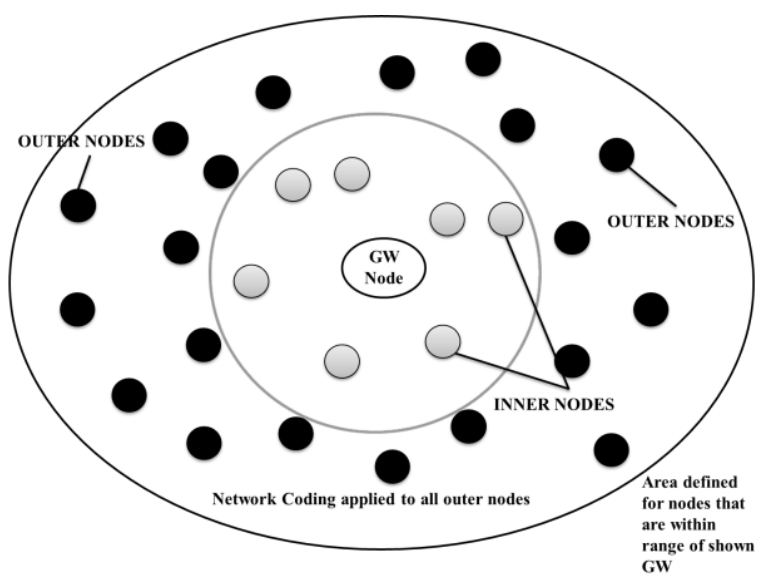

Fig. 3. WMN setup.

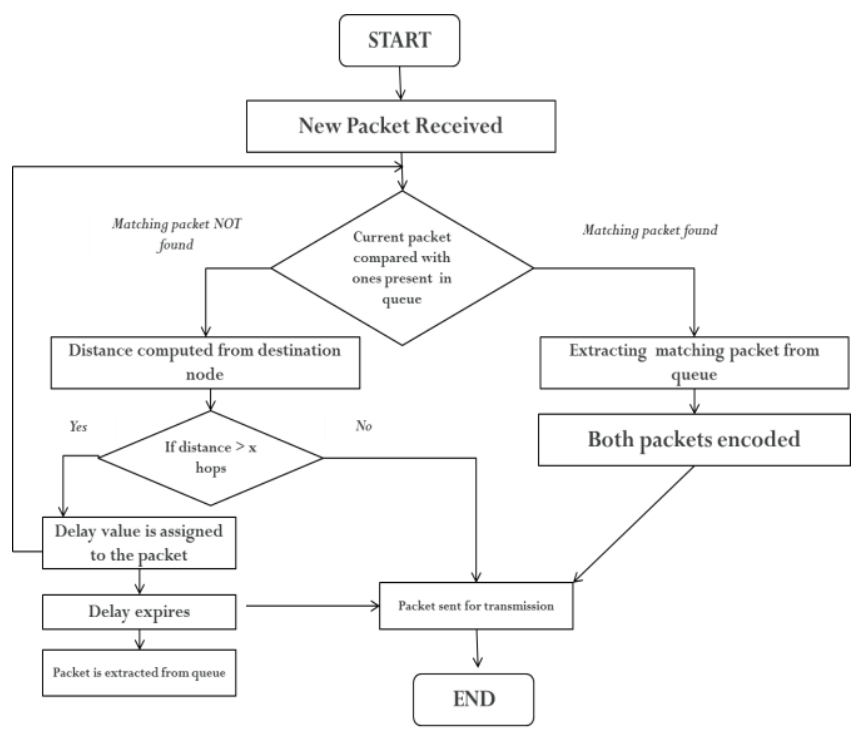

Fig. 4. Flowchart.

\section{ISSUE IDENTIFIED IN WMNS: CONGESTION}

Congestion occurs as a result of accumulation of traffic at particular nodes where the data queues gradually begin to build up. Hence as this congestion continues the performance of a network then begins to degrade.

Research in this area under the domain of wireless centralized networks and wired networks have been extensively studied in [10]-[12]. However in the context of WMNs whether these solutions can be applied or not has limited research [13], [14]. Similarly the congestion control solutions for centralized wireless networks cannot be applied due to the dynamic nature and distributed access control requirement in WMNs. Research done directly in the context of WMNs has been studied in [15]-[18].

In a WMN setup, the data is first traversed through its immediate neighbor nodes for routing. The data is then further sent to its next selected node until it reaches the specified node.

Within a WMN network data is being sent from the source node to its destination node; during this process the data being sent traverses through many intermediary nodes. Hence nodes can be simultaneously acting as source nodes as well as intermediary nodes in a WMN. The data at a given node will then be a combination of packets from all previous nodes of that particular route and its own generated data. Also from the gateway node data will be required to be sent to the requested nodes. Hence queues will begin to form at the gateway node and its nearby nodes. This scenario occurs recurrently in WMN and causes interference in achieving maximum potential of such networks.

Two categories of routing protocols were discussed, legacy based and diversity in literature survey. It is observed that a solution to the congestion problem can be solved by network coding from amongst the various routing protocols studied.

Network coding works on the principle that multiple packets can be sent in a single transmission. Similar to a coded packet that is a combination of a number of native packets, which after reaching the destination node will be decoded and the initial packets will be reconstructed. At the same time we will utilize the minimum time taken path between entree points and end users to our advantage along with network coding for the issue identified.

A brief description of network coding is given as follows.

\section{Network Coding}

A significant reduction in the number of transmissions can be achieved by employing network coding principles in a network. As an example we can illustrate that data is being transmitted between two nodes A and B; via a common relay node $\mathrm{R}$. Upon reception of respective data packets $\mathrm{pA}$ from node $\mathrm{A}$ and $\mathrm{pB}$ from node $\mathrm{B}$; an XOR operator is applied to both the packets and resulting packet is then broadcast to both the nodes. At the nodes the respective intended data packets can be recovered with analogous operations.

Via Network coding, the number of transmissions is reduced effectively from four transmissions using legacy routing to three transmissions in total, resulting in a network capacity increase of $33 \%$.

These packets often occur in WMNs particularly close to the gateway nodes. Identification of these packets and taking advantage of the possibility of applying Network coding on them results in decreasing the congestion that occurs in this scenario.

\section{PROposed AlgorithM}

The algorithm for network coding that has been modified is a general algorithm which can be applied to WMNs. Following is a brief explanation of the algorithm along with the modification introduced in order to cater to the issue of congestion identified in WMNs as shown in Fig. 3. In the proposed solution the shortest path between access points and end users is utilized along with network coding for the issue identified. In this context, an algorithm is proposed in which a timer is introduced in the network coding algorithm 
proposed

which will be able to optimize the network coding principle and hence produce better results. The value of the timer depends on the node's distance from the Gateway node within a network, hence the timer will be implemented only if the node is at $x$ no. of hops away from the Gateway node. Where $x$ can be varied according to size of the network. By applying the timer the opportunity or chance of implementation of network coding at that node increases, whereas by not applying a timer on every node within the network we will avoid unnecessary delay in the network is avoided that would hinder the performance at the end nodes and may initiate packet loss recovery mechanisms.

The Algorithm proposed is as follows:

\section{New Packet is received at a Node}

Step 1. New packet arrives at a Node.

Step 2. Comparison of received packet with existing packets in queue of Node is done.

Step 3. Upon packet matching the Node will extract that packet from the queue.

Step 4. Both packets are encoded by the Node.

Step 5. The encoded packet is now transmitted.

Step 6. If a match is not found THEN:

The node computes the distance of this packet from its destination node.

- If the computed distance is greater than $\mathrm{x}$ hops, a timer function will be added according to the value of which the packet will be delayed, this delayed packet will be added in the queue until the time for the timer is not equivalent to 0 .

- If the computed distance is equivalent to $\mathrm{x}$ hops or less than $\mathrm{x}$ hops, the packet is sent for transmission.

\section{Timer of Packet Expires}

Step 1. Duration of Timer attached to a packet ends.

Step 2. Packet is removed from queue by Node.

Step 3. The packet is now transmitted.

Flowchart for proposed algorithm is shown in Fig. 4. In basic network coding once a packet arrives at a node a pair able packet must be found that it can be encoded with this can be achieved by traversing each queue of the intermediary nodes that the data packet traverses before reaching its destination node. However in the case that no matching packet was found the packet will reach its destination in its original form without any coding performed on it. One method that can be employed in order to improve the possibility of packets being able to find their pair able packets is to delay a packet at each intermediary node with the chance that in that time interval a packet enters the queue that is pair able. Hence we apply a timer at each node; this method of using a timer before forwarding a packet is used in opportunistic routing mechanisms such as ExOR, SOAR and XCOR. An observation can be made that applying timers at each node will create a significant delay in the transmission of the packet therefore in the algorithm proposed the timer is attached to only nodes that are at a distance $\mathrm{x}$ hops from the destination. As an example if we observe the WMN set as shown in Fig. 3 where the Gateway node is the destination node then the timer is applied to only those nodes that are nearby to the Gateway node. This value of $x$ hops will be decided based on how far away a node is from its destination and nearby nodes will be chosen on the basis that the congestion area exists around the gateway since all data is being transmitted to and from it.

The flow chart shown in Fig. 4 is an overview of how data arriving at the source node is processed before transmission. For a packet to be directly transmitted a matching packet has to be found in the queue of packets. But in the case where a matching packet is not found in the queue against the incoming packet, the packets distance is computed from the destination node. If the distance is not greater than $\mathrm{x}$ hops, which is a defined threshold than the packet is sent for transmission otherwise a delay value is assigned to the packet and when the delay value expires it is sent for transmission and the packet is removed from the queue.

\section{CONCLUSION}

The main goal of this paper was to create a routing solution for congestion in a WMN, from the literature survey and taxonomy of routing protocols established for WMNs; it was observed that one solution to this problem can be achieved via Network coding. Network coding in WMN situations will lead to a better performance for solving congestion in these networks. In order to authenticate this we brought forward and carried out a modified Network Coding algorithm. Network Coding in WMN settings aims to address the congestion at transit links. Network Coding works on transit traffic at a node and attempts to increase its forwarding capability; thereby reducing data in the queues and resulting in lesser packet drops.

For future work the newly proposed algorithm can be explored in following factors:

- Implementation of proposed algorithm in a selected simulator such as NS2 or OPNET.

- The value of distance from source to destination can be varied and analyzed for an optimum value selection in various settings.

- Timer value can be optimized by analyzing in various WMN setups.

- Algorithm can be improved for different scenarios.

\section{REFERENCES}

[1] F. P. Kelly, "Models for a self-managed internet," Philosophical Transactions of the Royal Society, vol. 358, pp. 2335-2348, 2000.

[2] S. Kunniyur and R. Srikant, "End-to-end congestion control: utility functions, random losses and ECN marks," in Proc. the IEEE Infocom, Tel Aviv, vol. 3, March 2000, pp. 1323-1332.

[3] F. Paganini, J. Doyle, and S. Low, "Scalable laws for stable network congestion control," in Proc. the IEEE Conference on Decision and Control, December 2001, vol. 1, pp. 185-190.

[4] G. Holland and N. H. Vaidya, "Analysis of TCP performance over mobile ad hoc networks," in Proc. the IEEE/ACM Mobicom, August 1999, pp. 219-230.

[5] Y. Yan, B. Zhang, J. Zheng, and J. Ma, "CORE: Acoding aware opportunistic routing mechanism for wireless mesh networks," IEEE Wireless Communications, vol. 2, 2010.

[6] I. F. Akyildiz, X. Wang, and W. Wang, "Wireless mesh networks: A survey," Computer Networks, vol. 47, pp. 445-487, 2005. 
[7] R. Bruno, M. Conti, and E. Gregori, "Mesh networks: Commodity multihop adhoc networks," IEEE Communications Magazine, vol. 43 , pp. 123-31, 2005.

[8] S. Waharte, R. Boutaba, Y. Iraqi, and B. Ishibashi, "Routing protocols in wireless mesh networks: challenges and design considerations," Springer Science + Business Media, 2006.

[9] M. E. M. Campista, P. M. Esposito, I. M. Moraes, L. H. M. K. Costa, O. C. M. B. Duarte, D. G. Passos, C. V. N. de Albuquerque, D.C. M. Saade, and M. G. Rubinstein, "Routing metrics and protocols for wireless mesh networks in IEEE network," A Survey on Wireless Ad Hoc Networks, pp. 1-13, 2008.

[10] P. Gevros, J. Crowcroft, P. Kirstein, and S. Bhatti, "Congestion control mechanisms and the best effort service model," IEEE Network, vol. 15 , pp. 16-26, 2001.

[11] C. Q. Yang and A. V. S. Reddy, "Taxonomy for congestion control algorithms in packet switching networks," IEEE Network, vol. 9, pp. 34-45, 1995.

[12] Y. Su, "Congestion control in wireless multi-hop networks," Doctoral thesis, Swiss Federal Institute of Technology Zurich, 2008.

[13] T. Liu and W. Liao, "On routing in multichannel wireless mesh networks: Challenges and solutions," IEEE Network, 2008.

[14] S. Rangwala, A. Jindal, K. Jang, K. Psounis, and R. Govindan, "Understanding congestion control in multi hop wireless mesh networks," MobiCom, 2008.
[15] N. Nandiraju, D. Nanadiraju, L. Santhanam, B. He, J. Wang, and D. P P. Agrawal, "Wireless mesh networks: current challenges and future directions of web in the sky," IEEE Wireless Communications, 2007.

[16] Y. Bejerano, S. Han, and A. Kumar, "Efficient Load Balancing routing for Wireless Mesh Networks," Computer Networks, pp. 2450-2466, 2007.

[17] W. Song and X. Fang, "Routing with congestion control and load balancing in wireless mesh networks," in Proc. the $6^{\text {th }}$ International Conference on ITS Telecommunications Proceedings, 2006.

[18] L. Ma, "Load balancing and fair scheduling in wireless mesh networks," Master thesis, 2008.

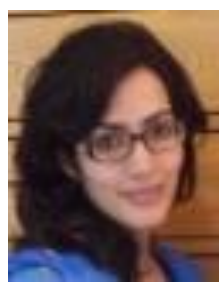

Sehrish Shoaib completed her bachelor in information and communication system engineering degree from National University of Sciences and Technology, Islamabad, Pakistan in 2008. She is currently enrolled in the Graduate Program of Computer Engineering with specialization in wireless networks from National University of Sciences and Technology, Islamabad, Pakistan. She remained a lab engineer at HITEC University, Taxila, Pakistan from 2008 to 2010, then joined National University of Sciences and Technology, Electrical and Mechanical College, Islamabad, Pakistan in the same capacity from 2010 to 2011. She also worked at IBM, Islamabad, Pakistan as a global support executive from 2011 to 2012. Ms. Shoaib is also a member of Pakistan Engineering Council (PEC). 